\title{
Measurement of the Diameter of the Common Bile Duct and Pancreatic Duct among Healthy Adult Sudanese Subjects using Magnetic Resonance Cholangiopancreatography
}

\author{
Rani Abu Elgasim, $\mathrm{MSc}^{1}$; Ahmed Abukonna, $\mathrm{PhD}^{1}$; Ala M. A. Elgyoum, $\mathrm{PhD}^{2}$; \\ Mogahid M. A. Zidan, $\mathrm{PhD}^{2}$; Mustafa Z. Mahmoud, $\mathrm{PhD}^{3 *}$; Moram A. Fagiry, $\mathrm{MSc}^{3}$; \\ Hamid Osman, $\mathrm{PhD}^{4}$; Mohamed Adam, $\mathrm{PhD}^{5}$ \\ ${ }^{1}$ Diagnostic Radiologic Technology Department, College of Medical Radiologic Sciences, Sudan \\ University of Science and Technology, Khartoum, Sudan \\ ${ }^{2}$ Diagnostic Radiology Department, Faculty of Radiological and Nuclear Medicine Science, National Ribat \\ University, Khartoum, Sudan \\ ${ }^{3}$ Radiology and Medical Imaging Department, College of Applied Medical Sciences, Prince Sattam bin \\ Abdulaziz University, Al-Kharj, Saudi Arabia \\ ${ }^{4}$ Department of Radiological Sciences, College of Applied Medical Science, Taif University, \\ Ta'if, Saudi Arabia \\ ${ }^{5}$ Radiological Sciences Department, College of Applied Medical Sciences, King Khalid University, \\ Abha, Saudi Arabia
}

\begin{abstract}
The purpose of our study was to evaluate the common bile duct (CBD) and pancreatic duct (PD) diameter among healthy adult Sudanese subjects using magnetic resonance cholangiopancreatography (MRCP). In addition, this study aimed to determine the effects of age, gender, and body height and weight on the CBD and PD diameters to establish a reference range for these ducts on MRCP, which is very useful in a daily clinical setting where MRCP is commonly performed to evaluate suspected biliary tract disease.

Methods and Results: This study included 80 asymptomatic subjects who underwent MRCP. The widest diameter of the CBD and PD was measured perpendicular to their long axes using the electronic caliper. The age, gender, medical history, body height and body weight were recorded.

Among the 80 subjects, the mean CBD diameter on MRCP was $6.17 \pm 0.69 \mathrm{~mm}$ (range of 4-8 $\mathrm{mm}$ ). There was a significant correlation between the CBD diameter and weight $(\mathrm{r}=0.407, P<0.001)$. The mean PD diameter on MRCP was $3.80 \pm 0.50 \mathrm{~mm}$ (range of 2-5 mm). There was also a significant correlation between the PD diameter and weight $(\mathrm{r}=0.407, P<0.001)$. In the cohort of 80 subjects, the mean CBD diameter in females was larger than in males $(6.50 \pm 0.632 \mathrm{~mm}$ and $5.95 \pm 0.677 \mathrm{~mm}$, respectively) $(P<0.05)$. Also, the mean PD diameter in females was statistically larger than in males $(6.03 \pm 0.66 \mathrm{~mm}$ and $5.58 \pm 0.675 \mathrm{~mm}$, respectively $)$ $(P<0.05)$. Our results demonstrate no significant correlation between the diameter of CBD and PD and participants' height and age.

Conclusion: The importance of the current study lies in it's being one of the few studies whose intention was to use MRCP to bridge the knowledge gap in the literature about the measurement of the CBD and PD diameter among healthy adult Sudanese subjects. (International Journal of Biomedicine. 2020;10(4):392-396.)
\end{abstract}

Key Words: magnetic resonance cholangiopancreatography $\bullet$ common bile duct $\bullet$ pancreatic duct

\section{Abbreviations}

CBD, common bile duct; CT, computed tomography; PD, pancreatic duct; MRCP, magnetic resonance cholangiopancreatography; BMI, body mass index. 


\section{Introduction}

The size of the common bile duct (CBD) is a predictor of biliary obstruction; in this connection an accurate CBD size reference range should be available. ${ }^{(1)} \mathrm{A}$ large number of published studies are devoted to the normal size of the CBD; however, an accurate reference range for CBD size remains debatable. ${ }^{(2-5)}$ The availability of an accurate reference range for CBD size would help to distinguish obstructive from nonobstructive causes of jaundice.

Today, MRCP is one of many newer noninvasive tests for assessment of biliary tract abnormality — especially those involving obstruction. ${ }^{(1,5)}$ MRCP provides information on both ductal and parenchymal features of cholangiopancreatography, such as $T_{1}$ signal changes, focal or diffuse gland atrophy, and irregular contour of the pancreatic duct (PD) ${ }^{(6)}$

The diameter of the CBD changes in response to various factors, including age, cholecystectomy, measurement location, respiration, and BMI..$^{(1,3,4,7-9)}$ In 1984, Wu et al., ${ }^{(10)}$ using real-time ultrasonography, found that the inner diameter of the widest point of the CBD varied from $1 \mathrm{~mm}$ to $10 \mathrm{~mm}$, and was found to be age-dependent $(\mathrm{r}=0.60, P<0.001)$. Later, other studies supported this observation. ${ }^{(1,3,4,7)}$ However, Horrow et al. ${ }^{(11)}$ obtained controversial results by routine abdominal sonography; they did not find an association between age and size of the extrahepatic bile duct (EHD) in 258 asymptomatic adults. Performing an ultrasound on 1484 normal individuals, Matcuk et al. (2) reported a small increase in EHD diameter with age $(+0.02 \pm 0.11 \mathrm{~mm} / \mathrm{y}, P<0.001)$ and female gender $(+0.3 \pm 1.6 \mathrm{~mm}, P<0.0001)$. However, two studies have suggested that gender has no significant effect on the $\mathrm{CBD}$ diameter by ultrasound and $\mathrm{MRCP}^{(1,4)}$

The purpose of our study was to evaluate the CBD and PD diameter among healthy adult Sudanese subjects using MRCP. In addition, this study aimed to determine the effects of age, gender, and body height and weight on the CBD and PD diameters to establish a reference range for these ducts on MRCP, which is very useful in a daily clinical setting where MRCP is commonly performed to evaluate suspected biliary tract disease.

\section{Materials and Methods}

The current study adhered to the Declaration of Helsinki and Title 4, US Code of Federal Regulations, Part 46, Protection of Human Subjects. This study was approved by the ethics committee of the College of Medical Radiological Sciences (CMRS), Sudan University of Science and Technology (Khartoum, Sudan). Written informed consent was obtained from each patient.

During the period of October 2019 to July 2020, 80 subjects (40 male and 40 female) aged between 24 and 95 years were enrolled in the study. The age, gender, medical history, list of medications, total serum cholesterol and liver function tests were documented for all subjects. In addition, body height and body weight were also recorded. Subjects with pre-existing hepatobiliary and pancreatic surgery, intra or retroperitoneal tumors, inflammation or hemorrhagic diseases, biliary tract stones, cholecystitis, cirrhosis of the liver, ascites or abnormal liver function tests were excluded from the current study.

MRCP examinations were performed using a 1.5 Tesla Toshiba MRI scanner with $120 \mathrm{mT} / \mathrm{m}$ maximum gradient capability and phase array body coil. MRCP examination was performed on patients after an overnight fast of at least 12 hours prior to the examination. After localizing images, the coronal and axial abdominal images were obtained using $\mathrm{T}_{2}$-weighted pulse sequences. Axial images of the biliary and pancreatic ducts were obtained using a $\mathrm{T}_{2}$-weighted, fat suppressed pulse sequence. In all subjects, MRCP included a breath-hold, thick-slab, single-shot turbo spin echo (ssTSE $\mathrm{BH})$ sequence images. The parameters of the ssTSE BH sequence were as follows: repetition time (TR) of $3137 \mathrm{msec}$; effective echo time (TE) of $512 \mathrm{msec}$; echo train length (ETL) or turbo factor of 128; flip angle of $90^{\circ}$; slice thickness of 30$40 \mathrm{~mm}$; field of view (FOV) of $250 \times 250 \mathrm{~mm}^{2}$; matrix size of $256 \times 205$; acquisition time of $8 \mathrm{sec}$. The same pulse sequence was repeated to acquire 4-6 projections of the pancreatic biliary system from different angles.

Measurements of the CBD and PD diameters were performed independently by an experienced radiological technologist with 20 years of experience with MR techniques. Coronal ssTSE BH images at 4-6 different angular planes were reviewed, and the one with well-demonstrated CBD and $\mathrm{BD}$ with the least superimposed artifacts was used for measurement. The widest diameter of the CBD and PD was measured perpendicular to their long axes using the electronic caliper. The applied MRCP imaging technique was in line with the guidelines used by Chen et al. ${ }^{(12)}$

Statistical analysis was performed using the statistical software package IBM SPSS Statistics for Windows, Version 22.0 (Armonk, NY: IBM Corp.). Continuous variables were presented as mean \pm standard deviation (SD). Student's unpaired t-test was used to compare two groups for data with normal distribution. Multiple comparisons were performed with one-way ANOVA. Pearson's correlation coefficient (r) was used to determine the strength of the relationship between the two continuous variables. A value of $P<0.05$ was considered significant.

\section{Results}

Among the 80 subjects, the mean CBD diameter on MRCP was $6.17 \pm 0.69 \mathrm{~mm}$ (range of 4-8 mm) (Table 1). There was a significant correlation between the CBD diameter and weight ( $r=0.407, P<0.001)$ (Fig. 1). On the other hand, the mean PD diameter on MRCP was $3.80 \pm 0.50 \mathrm{~mm}$ (range of 2-5 $\mathrm{mm})$. There was also a significant correlation between the PD diameter and weight ( $r=0.407, P<0.001)$ (Fig. 2). Furthermore, our results demonstrate no significant correlation between the CBD and PD diameters and participants' height.

In the cohort of 80 subjects, the mean CBD diameter in females was larger than in males $(6.4 \pm 0.632 \mathrm{~mm}$ and $5.95 \pm 0.677 \mathrm{~mm}$, respectively), and this difference was statistically significant $(P<0.01)$. Also, the mean PD diameter in females was statistically larger than in males $(4.03 \pm 0.460$ 
$\mathrm{mm}$ and $3.58 \pm 0.475 \mathrm{~mm}$, respectively), and this difference was statistically significant $(P<0.001)$ (Table 2$)$.

Table 1.

Descriptive statistic of study variables

\begin{tabular}{|l|c|c|c|c|}
\hline \multicolumn{1}{|c|}{ Variables } & Mean & SD & Minimum & Maximum \\
\hline Age (years) & 57.33 & 17.098 & 24 & 95 \\
\hline Weight $(\mathrm{kg})$ & 83.69 & 9.726 & 63 & 105 \\
\hline Height $(\mathrm{cm})$ & 167.25 & 10.06 & 140 & 180 \\
\hline CBD diameter $(\mathrm{mm})$ & 6.17 & 0.689 & 4.0 & 8.0 \\
\hline PD diameter $(\mathrm{mm})$ & 3.80 & 0.501 & 2.0 & 5.0 \\
\hline
\end{tabular}



Fig. 1. Correlation between CBD diameter and weight.

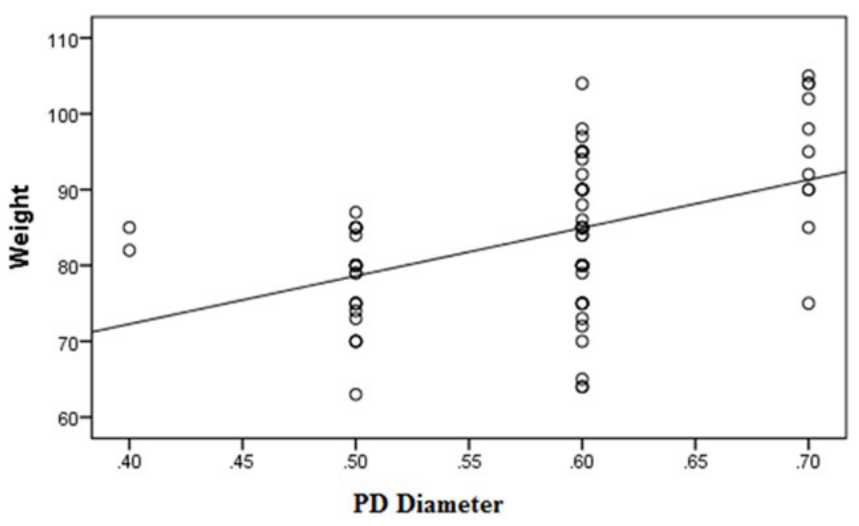

Fig. 2. Correlation between PD diameter and weight.

Results demonstrate the mean $\mathrm{CBD}$ and PD diameters of subjects in each age group (Table 3 ). The upper limit of the $95 \%$ reference range for the CBD diameter was 6.33 $\mathrm{mm}$, resulting in the reasonable upper limit of $6.5 \mathrm{~mm}$ for the asymptomatic subjects. There was no significant correlation between $\mathrm{CBD}$ diameters and age. For PD diameter, the upper limit of the $95 \%$ reference range was $4.05 \mathrm{~mm}$, resulting in the reasonable upper limit of $4 \mathrm{~mm}$ for the asymptomatic subjects. The PD diameter slightly increases until the age of 60 years, then decreases with an increase in age, but results were not statistically significant.

Table 2.

$C B D$ and $P D$ diameters in both genders

\begin{tabular}{|c|c|c|c|c|c|}
\hline Variables & Gender & $\mathrm{n}$ & Mean & SD & $P$-value \\
\hline \multirow{2}{*}{ CBD diameter $(\mathrm{mm})$} & Female & 40 & 6.4 & 0.632 & \multirow{2}{*}{0.0029} \\
\cline { 2 - 6 } & Male & 40 & 5.95 & 0.677 & \\
\hline \multirow{2}{*}{ PD diameter $(\mathrm{mm})$} & Female & 40 & 4.03 & 0.460 & \multirow{2}{*}{0.0001} \\
\cline { 2 - 6 } & Male & 40 & 3.58 & 0.475 & \\
\hline
\end{tabular}

Table 3.

Mean $C B D$ and $P D$ diameter in different age group

\begin{tabular}{|l|c|c|c|}
\hline \multicolumn{1}{|c|}{ Age group } & $\mathrm{n}$ & CBD diameter & PD diameter \\
\hline $20-30$ & 2 & $6.0 \pm 0.01$ & $3.50 \pm 0.51$ \\
\hline $30-40$ & 15 & $5.87 \pm 0.74$ & $3.80 \pm 0.58$ \\
\hline $40-50$ & 19 & $6.53 \pm 0.51$ & $3.89 \pm 0.54$ \\
\hline $50-60$ & 15 & $6.40 \pm 0.63$ & $3.93 \pm 0.51$ \\
\hline $60-70$ & 10 & $6.0 \pm 0.47$ & $3.70 \pm 0.48$ \\
\hline$>70$ & 19 & $6.0 \pm 0.82$ & $3.68 \pm 0.47$ \\
\hline Total & 80 & $6.17 \pm 0.69$ & $3.80 \pm 0.50$ \\
\hline
\end{tabular}

\section{Discussion}

In this study, the mean CBD diameter on MRCP was $6.17 \pm 0.69 \mathrm{~mm}$ (range of $4-8 \mathrm{~mm}$ ). Previous studies have shown that the mean CBD diameter was between 3.4 and 7.39 $\mathrm{mm}$, with a range of 1 to $15 \mathrm{~mm}$, and current results were well within the reported range. ${ }^{(1,4,7,13)}$ Our results for the upper limit of the $95 \%$ reference range for the CBD diameter $(6.5 \mathrm{~mm})$ are comparable to those from ultrasound and CT. ${ }^{(4,7)}$

A previous study has also suggested that body height and body weight have no significant effect on the CBD diameter. ${ }^{(3)}$ Daradkeh et al. ${ }^{(3)}$ reported that the CBD diameter was correlated with BMI by ultrasound. In our study, subjects' weight had a significant effect on the CBD diameter, but their height did not.

Several reports have considered the important agedependent variations in the CBD diameter. ${ }^{(1,3,4,7,13)}$ One study revealed a slight increase in duct diameter with advancing age. It has also been shown that the CBD diameter was directly proportional to age after patients were divided into 
two groups with 65 years as the cutoff age.(1) Park et al.(13) reported that the $\mathrm{CBD}$ diameter by $\mathrm{CT}$ in people over age 51 was significantly different from that in subjects under age 50 . However, Horrow et al. ${ }^{(11)}$ found no increase in the size of the extrahepatic bile duct with increasing age in an adult population. Our study supports the notion that age has no significant effect on the CBD diameter. Two other studies have reported that gender has no significant effect on the CBD diameter, ${ }^{(1,3)}$ which our findings also support. However, Matcuk et al. ${ }^{(2)}$ reported a small increase in EHD diameter with female gender.

Normal PD diameter in previous publications ranges from $2 \mathrm{~mm}$ to $8 \mathrm{~mm} .^{(14,15)}$ Our results for the mean PD diameter (range of 2-5 mm) were within the range. The PD diameter slightly increases until the age of 60 years, then decreases as age increases, but results were not statistically significant; nevertheless, they support the notion that increasing age causes atrophy of the pancreatic size. There was also a significant positive correlation between the PD diameter and body weight. These findings are in agreement with the previous studies. ${ }^{(16,17)}$

This study is limited by the unevenness of the population because of the randomized selection process, which unfortunately might affect the accuracy of our results and, in fact, significantly reduce the power of the conclusions because it makes other age groups have a lower statistical credibility if applied in future studies and may lead to some statistical bias. In addition, our screening questionnaires did not ask the participants for the list of medications that can cause CBD and PD dilatation, such as morphine, calcium antagonists, and nitroglycerine, as mentioned by Chen et al. ${ }^{(12)}$ and Economou et al. ${ }^{(18)}$ Despite the above limitations, the importance of the current study lies in it's being one of the few studies whose intention was to use MRCP to bridge the knowledge gap in the literature about the measurement of the CBD and PD diameter among healthy adult Sudanese subjects.

\section{Conclusion}

Our study shows that the average diameter of CBD and $\mathrm{PD}$ in the healthy adult Sudanese subjects, measured by MRCP, is $6.17 \pm 0.69 \mathrm{~mm}$ and $3.80 \pm 0.50 \mathrm{~mm}$, respectively. In addition, there was a significant correlation between the diameter of CBD and PD and a participants' weight. The mean diameter of CBD and PD in females was larger than in males, and this difference was statistically significant. Furthermore, our results demonstrate no significant correlation between the diameter of CBD and PD and participants' height and age.

\section{Acknowledgments}

This publication was supported by the Deanship of Scientific Research at Prince Sattam bin Abdulaziz University, Alkharj, Saudi Arabia.

\section{Competing Interests}

The authors declare that they have no competing interests.

\section{References}

1. Chen T, Hung CR, Huang AC, Lii JM, Chen RC. The diameter of the common bile duct in an asymptomatic Taiwanese population: measurement by magnetic resonance cholangiopancreatography. J Chin Med Assoc. 2012 Aug;75(8):384-8. doi: 10.1016/j.jcma.2012.06.002.

2. Matcuk GR Jr, Grant EG, Ralls PW. Ultrasound measurements of the bile ducts and gallbladder: normal ranges and effects of age, sex, cholecystectomy, and pathologic states. Ultrasound Q. 2014 Mar;30(1):41-8. doi: 10.1097/ RUQ.0b013e3182a80c98.

3. Daradkeh S, Tarawneh E, Al-Hadidy A. Factors affecting common bile duct diameter. Hepatogastroenterology. 2005 Nov-Dec;52(66):1659-61.

4. Senturk S, Miroglu TC, Bilici A, Gumus H, Tekin RC, Ekici F, Tekbas G. Diameters of the common bile duct in adults and postcholecystectomy patients: a study with 64 -slice CT. Eur J Radiol. 2012 Jan;81(1):39-42. doi: 10.1016/j. ejrad.2010.11.007.

5. Romagnuolo J, Bardou M, Rahme E, Joseph L, Reinhold C, Barkun AN. Magnetic resonance cholangiopancreatography: a meta-analysis of test performance in suspected biliary disease. Ann Intern Med. 2003 Oct 7;139(7):547-57. doi: 10.7326/0003-4819-139-7-200310070-00006.

6. Tirkes T, Shah ZK, Takahashi N, Grajo JR, Chang ST, Venkatesh SK, et al.; Consortium for the Study of Chronic Pancreatitis, Diabetes, and Pancreatic Cancer. Reporting Standards for Chronic Pancreatitis by Using CT, MRI, and MR Cholangiopancreatography: The Consortium for the Study of Chronic Pancreatitis, Diabetes, and Pancreatic Cancer. Radiology. 2019 Jan;290(1):207-215. doi: 10.1148/ radiol.2018181353.

7. Bowie JD. What is the upper limit of normal for the common bile duct on ultrasound: how much do you want it to be? Am J Gastroenterol. 2000 Apr;95(4):897-900. doi: 10.1111/j.1572-0241.2000.01925.x.

8. Wachsberg RH. Respiratory variation of extrahepatic bile duct diameter during ultrasonography. J Ultrasound Med. 1994 Aug;13(8):617-21. doi: 10.7863/jum.1994.13.8.617.

9. Peng R, Zhang L, Zhang XM, Chen TW, Yang L, Huang XH, Zhang ZM. Common bile duct diameter in an asymptomatic population: A magnetic resonance imaging study. World J Radiol. 2015 Dec 28;7(12):501-8. doi: 10.4329/ wjr.v7.i12.501.

10. Wu CC, Ho YH, Chen CY. Effect of aging on common bile duct diameter: a real-time ultrasonographic study. J Clin Ultrasound. 1984 Oct;12(8):473-8. doi: 10.1002/ jcu. 1870120804.

11. Horrow MM, Horrow JC, Niakosari A, Kirby CL, Rosenberg HK. Is age associated with size of adult extrahepatic bile duct: sonographic study. Radiology. 2001 Nov;221(2):411-4. doi: 10.1148/radiol.2212001700.

12. Chen T, Hung CR, Huang AC, Lii JM, Chen RC. The diameter of the common bile duct in an asymptomatic Taiwanese population: measurement by magnetic resonance

*Corresponding author: Professor Mustafa Z. Mahmoud, Radiology and Medical Imaging Department, College of Applied Medical Sciences, Prince Sattam bin Abdulaziz University, E-mail: m.alhassen@psau.edu.sa,PO Box: 422, Zip Code: 11942, Al-Kharj, Saudi Arabia. 
cholangiopancreatography. J Chin Med Assoc. 2012 Aug;75(8):384-8. doi: 10.1016/j.jcma.2012.06.002.

13. Park JS, Lee DH, Jeong S, Cho SG. Determination of Diameter and Angulation of the Normal Common Bile Duct using Multidetector Computed Tomography. Gut Liver. 2009 Dec;3(4):306-10. doi: 10.5009/gnl.2009.3.4.306.

14. Ladas SD, Tassios PS, Giorgiotis K, Rokkas T, Theodosiou P, Raptis SA. Pancreatic duct width: its significance as a diagnostic criterion for pancreatic disease. Hepatogastroenterology. 1993 Feb;40(1):52-5.

15. Hadidi A. Pancreatic duct diameter: sonographic measurement in normal subjects. J Clin Ultrasound. 1983 Jan;11(1):17-22. doi: 10.1002/jcu.1870110105.
16. Akochi S, Ugwu AC, Otuh I. Sonographic measurement of pancreatic duct diameter in apparently healthy adults in Abakaliki metropolis. International Journal of Sciences and Applied Research 2018;5(8):1-16.

17. Glaser J, Högemann B, Krummenerl T, Schneider M, Hultsch E, van Husen N, Gerlach U. Sonographic imaging of the pancreatic duct. New diagnostic possibilities using secretin stimulation. Dig Dis Sci. 1987 Oct;32(10):1075-81. doi: 10.1007/BF01300191.

18. Economou G, Ward-McQuaid JN. A cross-over comparison of the effect of morphine, pethidine, pentazocine, and phenazocine on biliary pressure. Gut. 1971 Mar;12(3):21821. doi: 10.1136/gut.12.3.218. 